\title{
Whole lung image-based quantification of alveolar airspace histological parameters identifies diffuse emphysematous phenotype in a mouse model of obstructive lung diseases
}

\author{
Ryunosuke Nakashima ${ }^{1}$, Hirofumi Nohara ${ }^{1,2}$, Shunsuke Kamei ${ }^{1,2}$, Haruka Fujikawa ${ }^{1,2}$, \\ Kasumi Maruta ${ }^{1}$, Taisei Kawakami ${ }^{1}$, Yuka Eto ${ }^{1}$, Mary Ann Suico ${ }^{1}$, Hirofumi Kai ${ }^{1}$, Tsuyoshi Shuto ${ }^{1}$ \\ ${ }^{I}$ Department of Molecular Medicine, Graduate School of Pharmaceutical Science, Kumamoto University, Japan, \\ ${ }^{2}$ Program for Leading Graduate Schools "HIGO (Health life science: Interdisciplinary and Global Oriented) Program", \\ Kumamoto University, Japan
}

$\underline{\text { Background }}$

Pulmonary emphysema is a pathophysiological characteristic of severe obstructive lung diseases including chronic obstructive pulmonary disease (COPD) and cystic fibrosis (CF). Recently, a murine model of COPD/CF that exhibits pulmonary emphysema has been uniquely established by inducing airway-specific overexpression of the epithelial $\mathrm{Na}^{+}$ channel $\beta$ subunit ( $\beta$ ENaC-Tg mice) (Shuto T., et al., Sci Rep, 2016). However, little is known about the biochemical and histological differences between $\beta \mathrm{ENaC}-\mathrm{Tg}$ mice and the existing emphysematous mouse model, for example, elastase-induced mouse model.

Method

We analyzed the biochemical parameters by quantitative RT-PCR using mouse lung tissue RNA samples. The histological assessment was performed employing a new method to evaluate emphysema in lung tissue section using whole lung image-based auto-measure parameters (area, perimeter, (major axis + minor axis) $/ 2$, feret diameter) by fluorescence microscope BZ-X710 (Keyence). We compared the parameters between $\beta \mathrm{ENaC}$-Tg mice and elastase-induced model mice, a general model of emphysema in COPD.

$\underline{\text { Results }}$

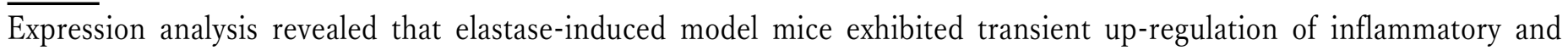
senescence-like markers (KC, IL-6, p21, Sirt1) at disease induction period (1 day after elastase administration), which did not last until onset of disease (after 3 weeks); while these up-regulations were stable and constant during the whole disease process in $\beta \mathrm{ENaC}$-Tg mice. Histological analyses revealed that even though the extent of emphysema was similar in both models, the coefficient of variation $(\mathrm{CV})$ of all alveolar airspace histological parameters was smaller in $\beta \mathrm{ENaC}-\mathrm{Tg}$ mice.

Conclusions

These assessments demonstrate that $\beta \mathrm{ENaC}$-Tg mice exhibit not only inflammatory and senescence-like but also diffusetype emphysematous phenotypes. The study also provides novel and useful image-based quantification method that covers whole lung tissue. 